\title{
Cysteine peptidases and their inhibitors in Tetranychus urticae: a comparative genomic approach
}

María Estrella Santamaría', Pedro Hernández-Crespo², Félix Ortego ${ }^{2}$, Vojislava Grbic ${ }^{1}$, Miodrag Grbic ${ }^{1}$, Isabel Diaz ${ }^{3}$ and Manuel Martinez ${ }^{3^{*}}$

\begin{abstract}
Background: Cysteine peptidases in the two-spotted spider mite Tetranychus urticae are involved in essential physiological processes, including proteolytic digestion. Cystatins and thyropins are inhibitors of cysteine peptidases that modulate their activity, although their function in this species has yet to be investigated. Comparative genomic analyses are powerful tools to obtain advanced knowledge into the presence and evolution of both, peptidases and their inhibitors, and could aid to elucidate issues concerning the function of these proteins.

Results: We have performed a genomic comparative analysis of cysteine peptidases and their inhibitors in T. urticae and representative species of different arthropod taxonomic groups. The results indicate: i) clade-specific proliferations are common to C1A papain-like peptidases and for the 125B cystatin family of inhibitors, whereas the C1A inhibitors thyropins are evolutionarily more conserved among arthropod clades; ii) an unprecedented extensive expansion for C13 legumain-like peptidases is found in T. urticae; iii) a sequence-structure analysis of the spider mite cystatins suggests that diversification may be related to an expansion of their inhibitory range; and iv) an in silico transcriptomic analysis shows that most cathepsin $B$ and $L$ cysteine peptidases, legumains and several members of the cystatin family are expressed at a higher rate in T. urticae feeding stages than in embryos.

Conclusion: Comparative genomics has provided valuable insights on the spider mite cysteine peptidases and their inhibitors. Mite-specific proliferations of C1A and C13 peptidase and 125 cystatin families and their overexpression in feeding stages of mites fit with a putative role in mite's feeding and could have a key role in its broad host feeding range.
\end{abstract}

Keywords: Comparative genomics, Cystatins, Cysteine peptidases, Feeding, Tetranychus urticae, Thyropins

\section{Background}

The two-spotted spider mite, Tetranychus urticae, is one of the main pests of agricultural crops due to its broad host range. This polyphagous species feeds on more than 1,100 plant species, from which about 150 are of great economic value. Thus, it represents a very important pest for field and greenhouse crops, ornamentals, annual and perennial plants all over the world. T. urticae has one of the smallest known animal genomes of about $90 \mathrm{Mbp}$ with over 18,000 genes identified. Genome

\footnotetext{
* Correspondence: m.martinez@upm.es

${ }^{3}$ Centro de Biotecnología y Genómica de Plantas, Universidad Politécnica de Madrid, Campus Montegancedo, 28223-Pozuelo de Alarcón, Madrid, Spain Full list of author information is available at the end of the article
}

features, such as large expansion of gene families associated with digestion and detoxification of plant secondary compounds are consistent with the spider mite's wide host feeding range [1].

Regarding mite digestive physiology, mites use both extracellular and intracellular digestion, with the latter occurring in gut wall-derived epithelial cells that ingest and digest food particles that can be free floating $[2,3]$. The midgut is the site for synthesis and secretion of digestive enzymes and absorption of nutrients. Processed food and epithelial cells pass into the posterior midgut, are subsequently compacted in the hindgut and excreted as faecal pellets [3]. Mite species that feed on plants rely

\section{Biomed Central}

(c) 2012 Santamaría et al.,licensee BioMed Central Ltd. This is an Open Access article distributed under the terms of the Creative Commons Attribution License (http://creativecommons.org/licenses/by/2.0), which permits unrestricted use, distribution, and reproduction in any medium, provided the original work is properly cited. 
mostly on cysteine peptidase activities for the digestion of dietary proteins $[4,5]$.

Cysteine peptidases are enzymes that hydrolyse peptide bonds using a catalytic cysteine. The MEROPS database contains all the existing peptidases grouped in clans [6]. Clans represent one or more families that show evidence of their evolutionary relationship by their similar tertiary structures, or when structures are not available, by the order of catalytic-site residues in the polypeptide chain and often by common sequence motifs around the catalytic residues. At present, among the 72 families of cysteine peptidases identified, 43 are included in 8 clans exclusively formed by cysteine peptidases, 13 are distributed in three clans that comprise peptidases with different catalytic mechanisms, and 16 are not enclosed in any determined clan. Most of the cysteine peptidases characterized in arthropods belong to the papain-like family $(\mathrm{C} 1 \mathrm{~A})$, although members of the legumain (C13), calpain (C2), caspase (C14) or separase (C50) families have also been reported.

Arthropod papain-like cysteine peptidases are homologous to mammalian cathepsins, which are present in lysosomes, but can also be localized in extracellular spaces [7]. In mites and insect species belonging to the orders Coleoptera, Hemiptera and Homoptera most C1A peptidases, particularly cathepsins L and B-like, are involved in the digestion process [8-10]. Besides, they are implicated in other physiological processes in arthropods, such as embryogenesis or metamorphosis [11-14]. For T. urticae, in vitro assays determined that major protease activity in extracts relies on papain-like cysteine type protease activity [4], which match up with the proliferation of this gene family in the spider mite genome [1]. In addition, a multigene family of legumain genes was also found in the spider mite genome [1], which could have a role in feeding similar to that observed for a legumain peptidase related to the digestive process of the hard tick Ixodes ricinus [15].

The most known inhibitors of $\mathrm{C} 1 \mathrm{~A}$ and $\mathrm{C} 13$ peptidases are the members of the I25 cystatin superfamily. In arthropods, the two ancestral eukaryotic lineages of the cystatin superfamily, stefins (I25A) and cystatins (I25B), have been reported [16]. Stefins are single copy intracellular inhibitors without disulphide bridges. Cystatins undergo frequent duplication events during evolution, and have a signal peptide and one or two disulphide bridges. The inhibitory consensus motif for C1A peptidases is formed by a conserved glycine residue in the N-terminal region, a QxVxG motif in the central region of the polypeptide and a tryptophan in the $\mathrm{C}$ terminal region [17], although proteins with variants in these motives are also active as inhibitors, such as the sialostatins from ticks that lack the conserved tryptophan residue [18]. The inhibitory activity against C13 peptidases is achieved by an asparagine included in the consensus motif $\mathrm{S} / \mathrm{T}-\mathrm{N}-\mathrm{D} / \mathrm{S}-\mathrm{M} / \mathrm{I} / \mathrm{L}$ based on vertebrate and plant cystatins $[19,20]$, although the ability to inhibit legumains by variants of this motif has not been tested. Some studies have suggested that cystatins from arthropods have functions related to the control of endogenous proteolysis, the balance of host-vector immune relationships, innate immunity, or antimicrobial defence [21-24]. In addition, two other types of cysteine peptidase inhibitors, the propeptide regions of $\mathrm{C} 1 \mathrm{~A}$ peptidases (I29) and the thyropins (I31) have also been reported in arthropods [25,26]. However, with the exception of the cystatins that contributes to blood feeding in ticks by suppressing host immune response [27], their potential role in the feeding process of arthropods has yet to be investigated.

Comparative genomic analyses provide valuable insights into the conservation and evolution of protein families, which could aid to elucidate issues concerning their function [28]. Thus, we have performed a comparative study of the gene families of cysteine peptidases and their inhibitors in representative species of different taxonomic groups belonging to Arthropoda. This analysis has been focused in those cysteine peptidases potentially involved in T. urticae feeding [4]. Results indicate mite-specific proliferations of $\mathrm{C} 1 \mathrm{~A}$ and $\mathrm{C} 13$ cysteine peptidases and their inhibitors I25B cystatins, as well as a correlation between the in silico expression of specific cystatins with putative digestive cysteine peptidases, providing evidences for their involvement in the feeding process of the spider mite.

\section{Results}

C1A and C13 cysteine peptidases and their inhibitors in fully sequenced arthropods

As previously described, a proliferation of both $\mathrm{C} 1 \mathrm{~A}$ and C13 cysteine peptidases was detected in the genome of the two-spotted spider mite [1]. To obtain further insights on the genomic content of proteins putatively related to the proteolityc digestive process of T. urticae, we extended the search of members from these peptidase families and their inhibitors to other species of arthropods, whose genomes have been completely sequenced and annotated, and drafts of these sequences are available on the web. The selected species were: two acari, T. urticae and Ixodes scapularis (black legged tick); one crustacean, Daphnia pulex (common water flea); and ten insect species, the dipterans Drosophila melanogaster (fruit fly) and Anopheles gambiae (African malaria mosquito), the lepidopteran Bombyx mori (domestic silkworm), the coleopteran Tribolium castaneum (red flour beetle), the hymenopterans Camponotus floridanus (carpenter ant), Apis mellifera (honey bee) and Nasonia vitripennis (jewel wasp), the hemipterans Rhodnius 
prolixus (kissing bug) and Acyrthosiphon pisum (pea aphid), and the phtirapteran Pediculus humanus corporis (human body louse).

The results obtained from genome extensive searches compared with the location of each species in the phylogenetic tree of arthropods are summarized in Figure 1. All the species have C1A cysteine peptidases, although the highest number of genes was found in T. urticae when compared with all the insect and even the other acari species analysed. Likewise, from the $\mathrm{C} 13$ family of peptidases, a strong proliferation of legumains was detected in T. urticae, 19 genes, against a maximum relative to the three genes encoding legumains in the herbivore pea aphid. On the contrary, a unique gene encoding a C13 GPI:protein transamidase was present in all species analysed. Differences in the number of cysteine peptidase genes could be hypothetically correlated with differences in the number of their putative inhibitors. Thus, we look for the gene content of both, the cystatins (I25CPI), which are putative inhibitors of $\mathrm{C} 1 \mathrm{~A}$ and $\mathrm{C} 13$ peptidases, and thyropins (I31Thy), which are putative inhibitors of C1A cysteine peptidases. As for C1A papains, the number of cystatins was considerably higher in T. urticae and in the crustacean $D$. pulex than in other insect species, whereas the number of thyropins was only slightly higher. Interestingly, I. scapularis, the other chelicerata species used in this study, showed a similar number of both cysteine peptidases and inhibitors than the insect species analyzed. As clade-specific proliferations were previously detected for $\mathrm{C} 1 \mathrm{~A}$ cysteine peptidases and C13 legumains [1], next analyses were focused to the evolutionary features of cysteine peptidase inhibitors.

\section{Gene content evolution of 125 cystatins in arthropods}

Arthropod members of the cystatin superfamily belong to the stefin and cystatin lineages. Stefins were not present in the insect species and one stefin encoding gene was found in the genomes of the mite T. urticae and in the crustacean D. pulex. Architectures for proteins containing domains of the cystatin lineage vary among different clades. Three different architectures were detected (Figure 2A). Proteins including a unique cystatin domain were only detected in the annotated genomes of the two species of acari analysed, the crustacean, the dipteran species, and in P. humanus. Multicystatin proteins containing only cystatin domains, whose number range from two to eight, were only present in $D$. pulex and $R$. prolixus. In contrast, multicystatin proteins (two to twelve domains) containing an additional C1A cysteine peptidase domain were found in the crustacean $D$. pulex and in all the insect species analysed, with the exception of $R$. prolixus. Interestingly, these multicystatin-C1A peptidase proteins were not present in the acari species.

To understand how the cystatin lineage has evolved in the different arthropod clades, the individual I25B

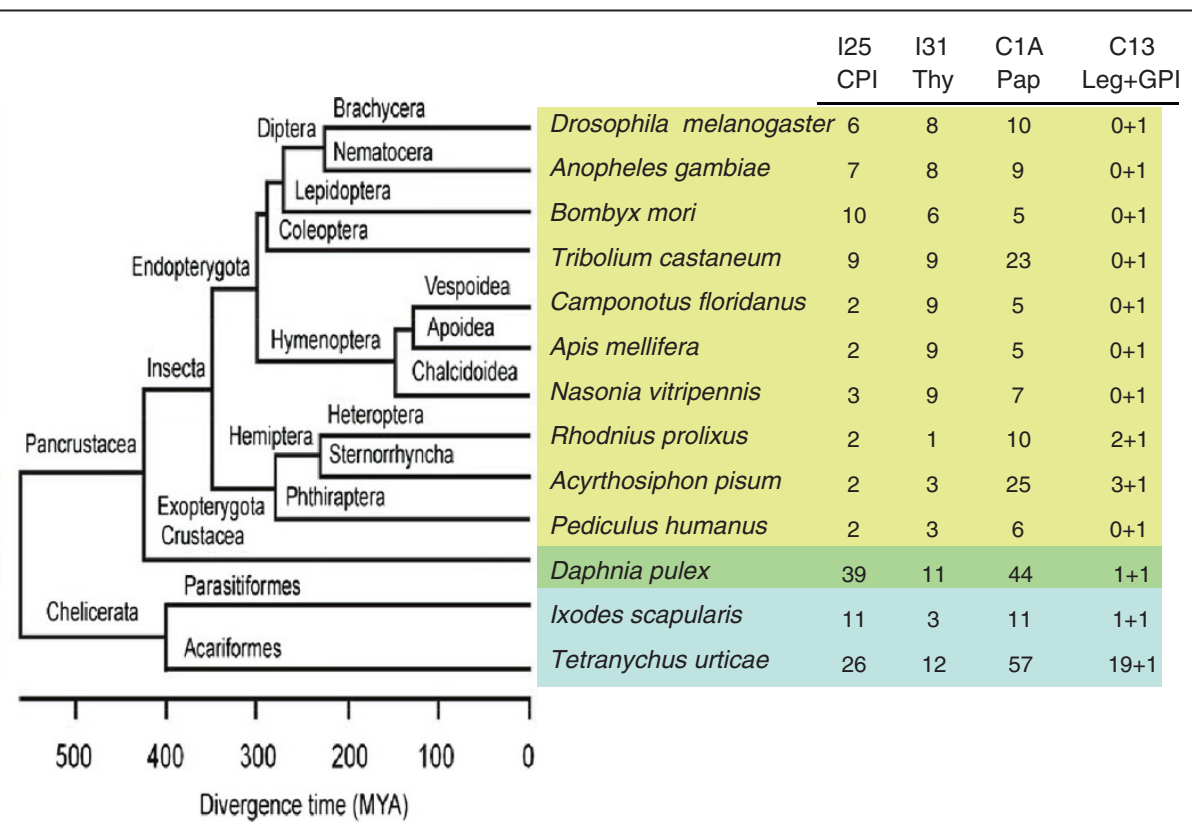

Figure 1 Number of cysteine peptidases and their inhibitors in selected arthropod species. Schematic evolutionary tree of fully sequenced arthropods including for each species the number of inhibitory cystatin (I25CPI) and thyropin (I31Thy) domains and the C1A (papain-like, C1APap) and C13 (legumain-like, C13Leg and GPI:protein transamidases, C13GPI) cysteine peptidases, putative targets of both inhibitors. MYA, million years ago. 


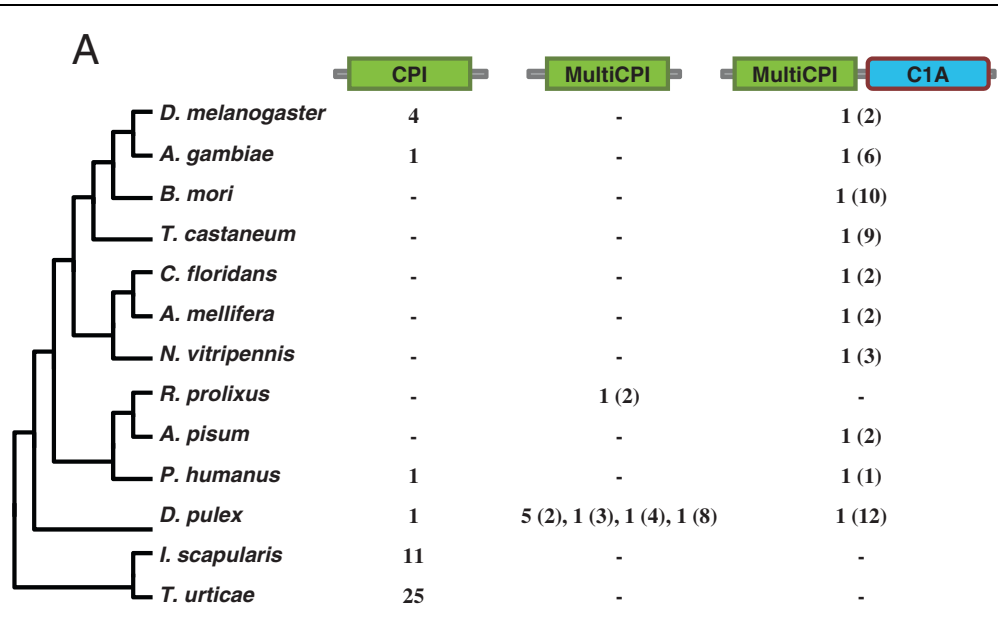

B

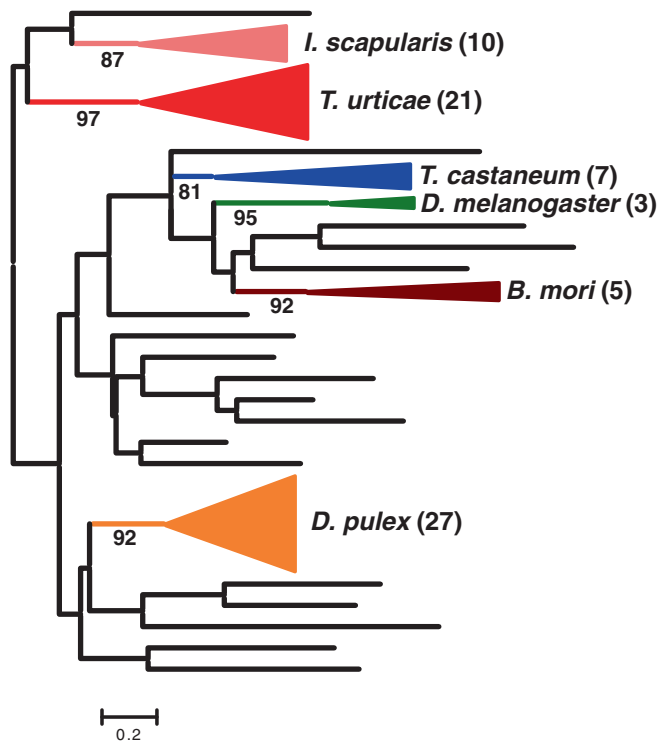

Figure 2 Evolutionary features of cystatins in arthropods. (A) Number of cystatin domains in each species distributed in base of their protein architecture. In brackets the number of repeats in multicystatin domains. CPI, single cystatin domain; MultiCPI, multicystatin domains. (B) Phylogenetic tree using the selected cystatin sequences from the different arthropod species. Coloured triangles show species-specific gene proliferations. In brackets the number of proteins in each subtree.

domains were aligned by MUSCLE (see Additional file 1A). Extensive amino acid differences avoid the construction of a robust phylogenetic tree using all the cystatin sequences. Thus, sequences contributing to extensive gaps in the conserved regions of the alignment were discarded and a phylogenetic tree constructed by the maximum likelihood PhyML method (see Additional file 2A). The corresponding schematic cladogram is shown in Figure 2B. As highlighted, clado-specific proliferations are detected, supported by approximate likelihood-ratio test values (aLRT) higher than $80 \%$. This cladogram suggests that the evolution of the cystatin family in arthropods is the result of extensive duplications from the ancestral genes probably determined for specific features in each clade.

\section{Gene content evolution of 131 thyropins in arthropods}

As for the cystatin family, we performed a phylogenetic analysis of the individual thyropin domains to know how this family has evolved in the different arthropod clades. The I31 domains were aligned by MUSCLE (see Additional file $1 \mathrm{~B}$ ), and a phylogenetic tree was constructed by the maximum likelihood PhyML method (see Additional file 2B), after discarding sequences contributing to extensive gaps in the conserved regions of the alignment. The corresponding schematic cladogram is shown in Figure 3A. A search for additional domains in proteins containing thyropin regions gives a broad combination of different domains (see Additional file 3). The repertoire of additional domains includes the serine peptidase inhibitory domains Kazal 2, Kunitz and Antistasin (Ant), the peptidase related 

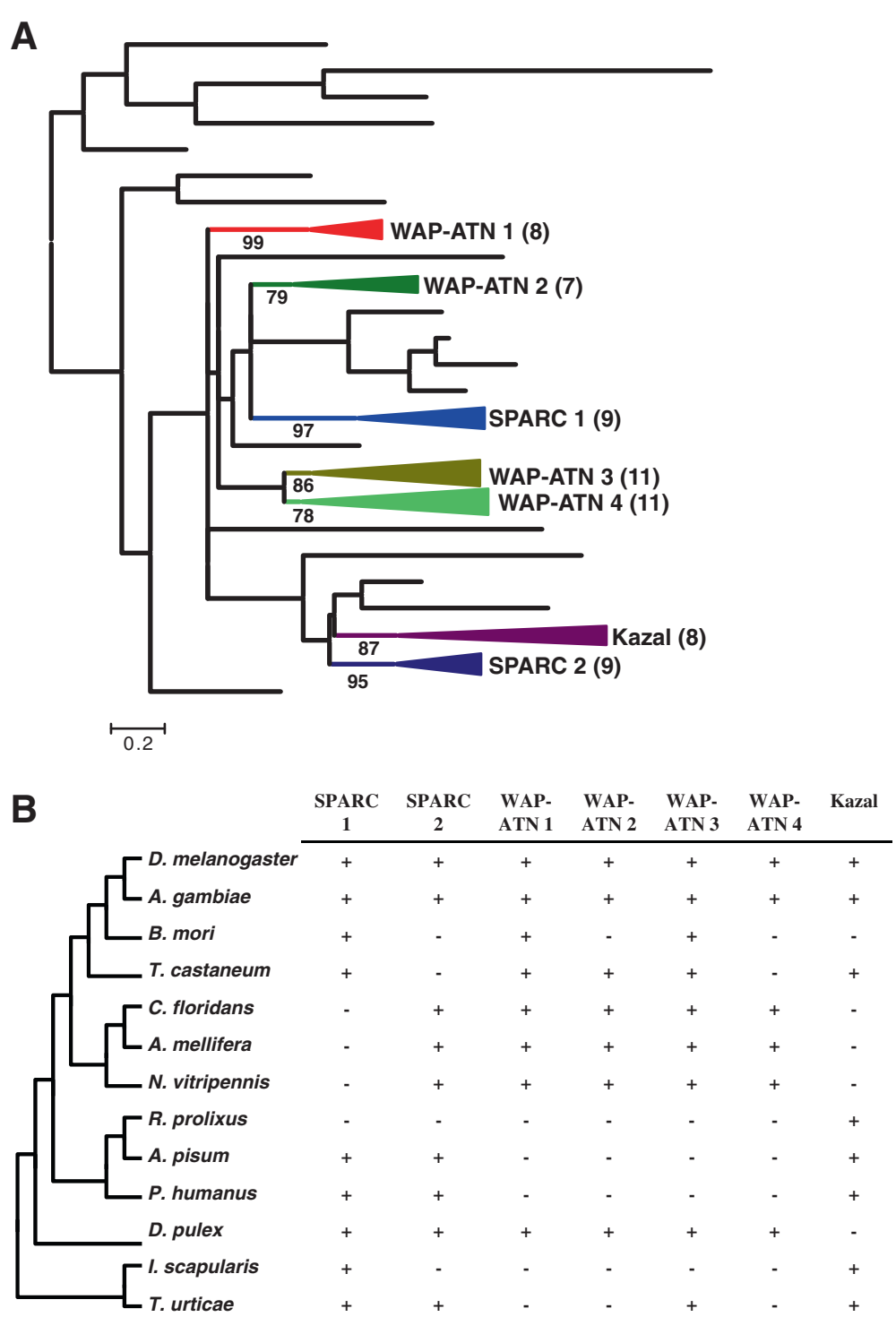

Figure 3 Evolutionary features of thyropins in arthropods. (A) Phylogenetic tree using the selected thyropin sequences from the different arthropod species. Coloured triangles show thyropin gene proliferations associated to other protein domains. In brackets the number of proteins in each subtree. (B) Presence (+) or absence $(-)$ of thyropins members of each subtree in the different arthropod species. WAP, whey acidic protein; ATN, antistasin; SPARC, secreted protein acidic and rich in cysteine.

inhibitory domain WAP (whey acidic protein), the calcium binding domain SPARC (secreted protein acidic and rich in cysteine), and the putative GTP-binding domain nuc121. As remarked, groups including thyropin domains associated to a second peptide domain and supported by approximate likelihood-ratio test values (aLRT) around or higher than $80 \%$ were found. When we analysed the composition of these groups, we observed that they are composed by proteins belonging to different arthropod species (Figure $3 \mathrm{~B}$ ). Proteins with thyropin domains associated to SPARC, WAP-ATN or Kazal domains are shared by insect, crustacean and acari species. A comparison of the cladograms showed in Figures $2 \mathrm{~B}$ and $3 \mathrm{~A}$ implies a different evolutionary process from cystatins and thyropins, with a small weight of clade-specific proliferations in the evolution of thyropins.

\section{Structural features of $T$. urticae cystatins}

Since the cystatin lineage had a clade-specific pattern of proliferations, they could be related to the inhibition of the expanded groups of $\mathrm{C} 1 \mathrm{~A}$ and $\mathrm{C} 13$ cysteine peptidases of $T$. urticae. Thus, we analysed in more detail the sequence-structure relationships in this family of proteins. To find how spider mite cystatins are grouped, their I25B domains were aligned by MUSCLE (see Additional file 1C), and a phylogenetic tree was constructed by the 
maximum likelihood PhyML method. Additionally, a representation of the alignment was done including the location of the key residues involved in cysteine peptidase inhibition, and of the secondary structures predicted in their tri-dimensional conformation (see Additional file 4). Four different groups were detected, which had specific features in their amino acid sequences (Figure 4A) and a different number of protein members. Whereas groups 1 and 3 consisted of 1 or 2 genes, groups 2 and 4 have been extensively expanded in restricted scaffold regions probably due to recent gene duplication events. Group 1 is formed by TuCPI-1, which is the only $T$. urticae cystatin sequence that have the consensus motifs for $\mathrm{C} 1 \mathrm{~A}$ peptidase inhibition: a $G$ in the amino acid part of the protein, the conserved QxVxG in the first loop and a W in the second loop. Besides, it has the consensus motif including the asparagine responsible to legumain inhibition as well as the four cysteine residues involved in disulphide bridges. Group 2 is constituted by cystatins TuCPI- 2 to $-6,-8,-15$, and -18 to -22 . Most members of this group retain the asparagine putatively involved in C13 inhibition surrounded by variants of the consensus inhibitory sequence, and all conserved motifs for $\mathrm{C} 1 \mathrm{~A}$ inhibition with the exception of the tryptophan in the second loop, which is replaced by several different amino acids. In contrast, most of these proteins have a conserved tryptophan residue four amino acids before the expected location of this. Group 3 is composed by TuCPI-7 and -12, and its members are similar to that of the second group, with the new conserved tryptophan residue, but they lack the cysteine residues that form the second disulphide bridge and the asparagine important for legumain inhibition. Finally, group 4 including TuCPI-9 to $-11,-13,-14,-16,-17$, and -23 to -25 , is the most striking clade. The proteins that belong to this group do not present the conserved residues involved in $\mathrm{C} 1 \mathrm{~A}$ inhibition, neither the $\mathrm{G}$ nor the QxVxG or any of the tryptophans in the C-terminal part of the molecule. However, they maintain the four cysteine residues and have several conserved motifs in their sequences, as an YNK motif after the putative $\alpha$-helix location. Besides, some of its members have an asparagine residue in the location where it is involved in legumain inhibition.

To determine how amino acid differences can influence the tri-dimensional structure of the cystatins, the structures of the TuCPI-1, $-3,-7$, and -13 proteins, representatives of each group, were modelled using the crystallographic structure of the cystatins from chicken egg (1YVBI), soft tick (3L0R), and human cystatin F (2CH9) (Figure 4B). TuCPI-1 and -7 aligned to chicken egg cystatin at sequence identities of $30 \%$ and $23 \%$, and with Q-MEAN Z-scores of -3.36 and -2.87 , respectively. TuCPI-3 and -13 aligned to soft tick cystatin and human cystatin $\mathrm{F}$ at sequence identities of $28 \%$ and $22 \%$, and with Q-MEAN Z-scores of -2.32 and -1.76 , respectively. These results imply relatively accurate models for T. urticae cystatins. From models, strong differences in the putative region for $\mathrm{C} 1 \mathrm{~A}$ peptidase inhibition were observed. The consensus amino acid residues in TuCPI1 fit with a canonical interaction with $\mathrm{C} 1 \mathrm{~A}$ peptidases. On the contrary, the conserved tryptophan in TuCPI-3 and -7 located at the end of the third $\beta$-sheet could be involved in a distinct interaction with these peptidases leading to changes in their inhibitory specificity. In the case of TuCPI-13, the lack of conserved residues in the domain responsible to $\mathrm{C} 1 \mathrm{~A}$ peptidase inhibition could mean a lack of inhibition to these peptidases. TuCPI-1 and -3 also have an asparagine in the loop after the conserved $\alpha$-helix that could be involved in legumain inhibition. This asparagine was absent in TuCPI-7 and -13 . However, four group cystatins have at least two different conserved motifs (YNK and SKPY) at the spatial region where the $\mathrm{C} 13$ inhibitory activity is achieved. A role for the asparagine of the YNK motif in legumain inhibition could be hypothesized, although the conserved motifs could be alternatively related to a different function.

\section{Expression profiling of cysteine peptidases and inhibitors}

To correlate genomic proliferations to gene expression, an in silico analysis of transcriptome expression was performed using the RNA-seq information available at the BOGAS T. urticae database. Most genes for cysteine peptidases and inhibitors had transcriptomic data. Only the stefin and seven cystatin genes had not RNA-seq information available. Figure $5 \mathrm{~A}$ shows the developmental mite stages in which the highest level of expression was detected for each gene analysed. Genes belonging to the C1A cathepsin L and B, C13 legumain and I25 cystatin groups were more expressed in the feeding stages of development, mainly in the adult phase. These genes had a wide expression range, having many genes expression values over 100 and even over 1000, but also lesser than 10 (Figure 5B). Furthermore, the comparison of the expression levels between adults and embryos showed that more than $75 \%$ of the cathepsin B-like, cathepsin L-like, legumain and cystatin genes with an expression value higher than 5 were significantly overexpressed in adults (Table 1). On the contrary, genes included in the $\mathrm{C} 1 \mathrm{~A}$ cathepsin O, C13 GPI:protein transamidase, C2 calpain, C14 caspase, C50 separase and I31 thyropin groups, did not show a specific developmental pattern (Figure 5A), having most of them a similar level of expression in all the mite stages analysed (data not shown), with normalised expression values among 10 and 100 (Figure 5B). Likewise, none of the genes for these families was significantly more expressed in adults than in embryos (Table 1).

A similar analysis was conducted for the four groups of spider mite cystatins. The different groups have 
A

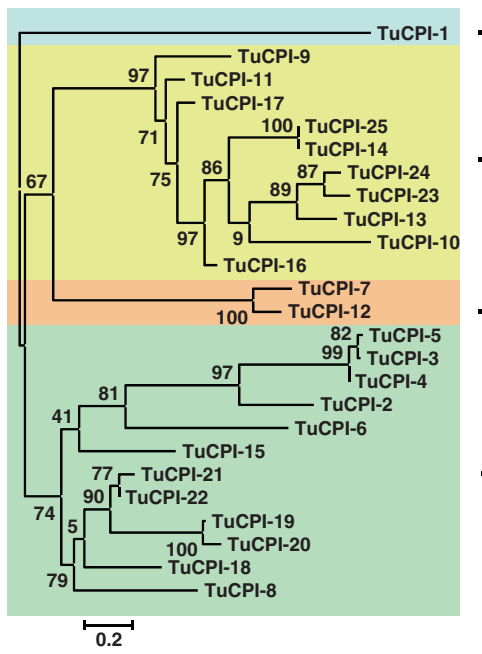

B
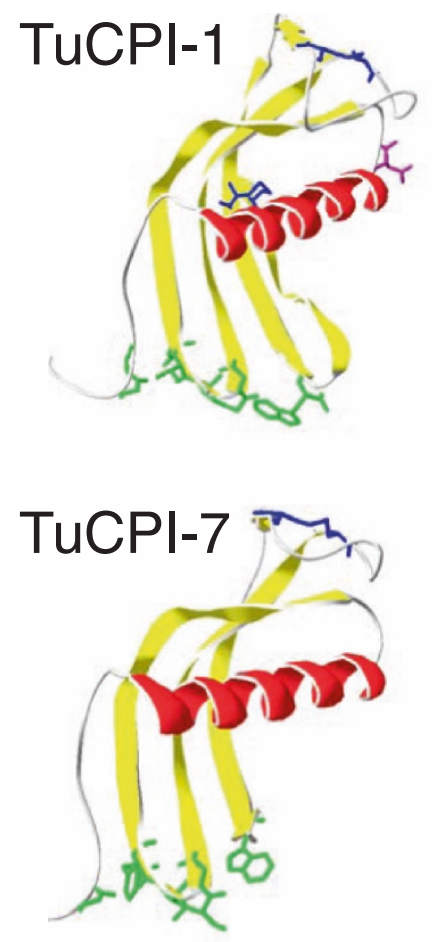

Group 1

G-N- $\mathrm{axV \times G}-\mathrm{C}-\mathrm{C}-\mathrm{C}-\mathrm{W}-\mathrm{C}-$

Group 4

$\mathrm{N}-\mathrm{YNK}-\mathrm{C}-\mathrm{C}-\mathrm{C}-\mathrm{C}-$

\section{Group 3}

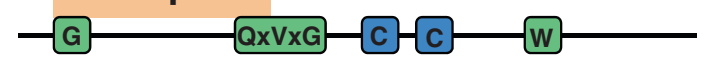

\section{Group 2}
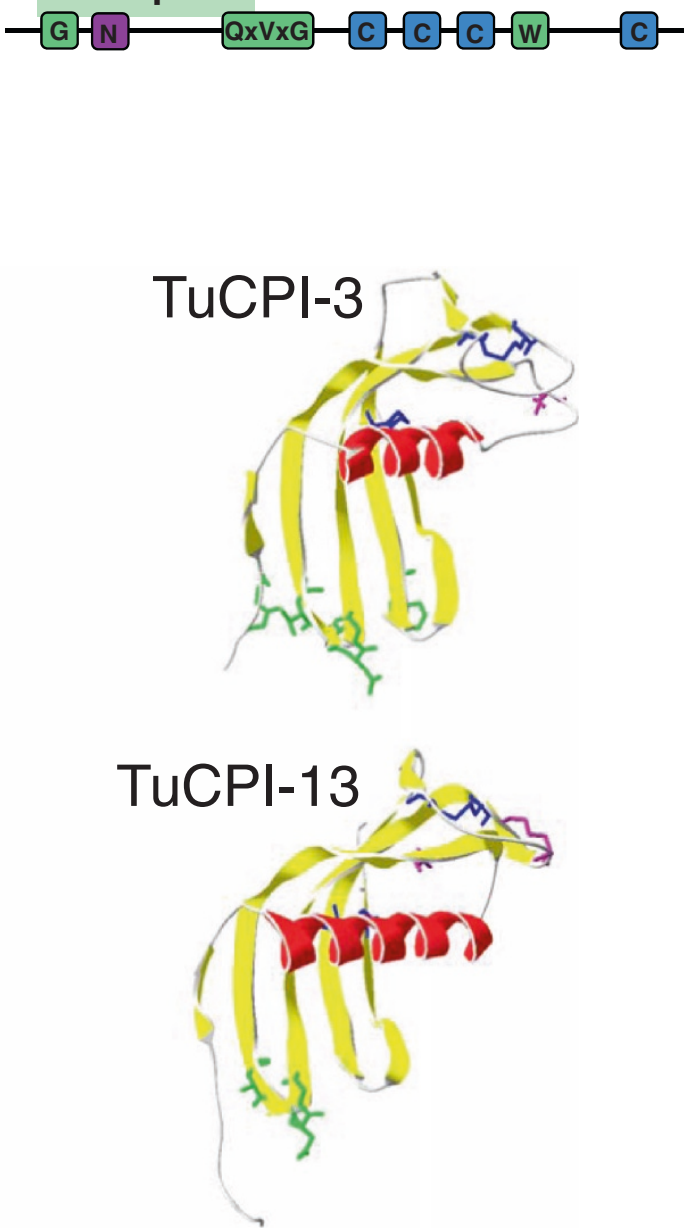

Figure 4 Evolutionary features of cystatins in T. urticae. (A) Phylogram of T. urticae cystatin sequences showing the presence of functional conserved residues in the four cystatin groups deduced from the phylogenetic tree. Amino acid sequences responsible of the inhibitor-C1A enzyme interaction are shaded in green. Putative sequences involved in C13 legumain inhibition are coloured in purple. Cysteine residues involved in disulphide bridges are in blue. (B) Homology models of T. urticae cystatins created using SWISS-MODEL. The residues in the region that interacts with C1A peptidases are coloured in green. The residues that could be involved in legumain inhibition are coloured in purple. Cysteines are coloured in blue. Red, a-helix; yellow, $\beta$-sheets.

specific expression patterns (Figure 5C, D). TuCPI-1, the only protein in group 1 , was highly expressed in all developmental stages (data not shown) with its maximum expression in nymphs. Members of group 2 showed the highest expression values and were most abundant in adults and nymphs. Conversely, group 3 cystatins presented the lower level of expression, with one gene mostly expressed in embryos and the other in larvae. 


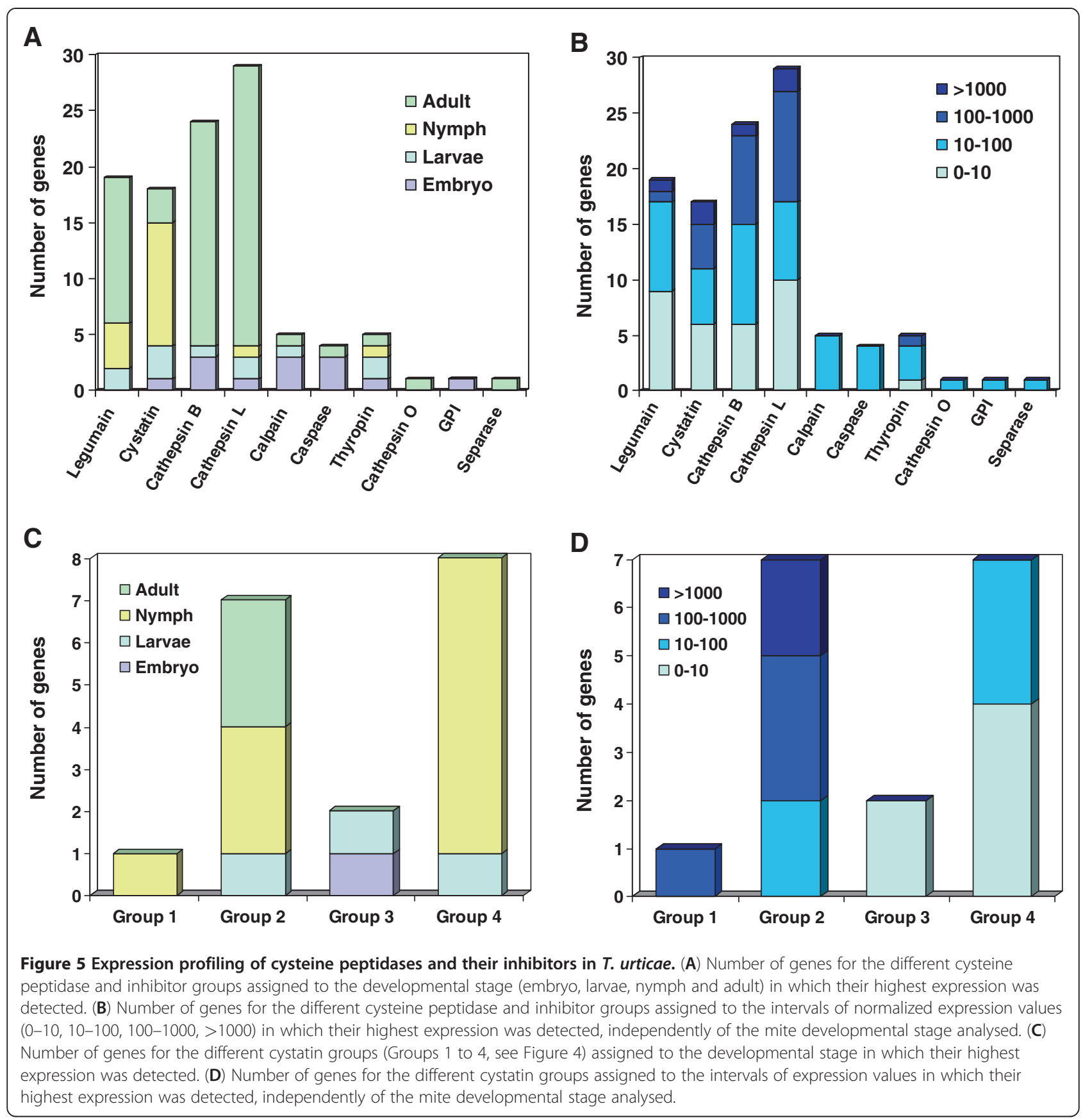

Finally, the members of group 4 had, in general, low expression values, but had an interesting developmental pattern, with a weaker expression in adults or embryos than in nymphs.

\section{Discussion}

Cysteine peptidases are crucial in different arthropod physiological processes [11-14], including the digestion of dietary proteins in mites and some insect species [8-10]. The dominant cysteine peptidase activity detected in body extracts of $T$. urticae points out their main role in proteolytic digestion after feeding [4,5]. In addition, the proliferation of $\mathrm{C} 1 \mathrm{~A}$ papain and $\mathrm{C} 13$ legumain families in the recently annotated genome of $T$. urticae and changes in expression detected in these peptidase genes after host change suggest their implication in the feeding process and, most probably, on the ability of mites to feed on the large number of plant hosts [1].

The broad multigene family of papain-like cysteine peptidases and the unusual proliferation of legumain peptidases in $T$. urticae were found to have no counterparts after extending the analysis to the annotated 
Table 1 Expression of cysteine peptidases and inhibitors in adults against embryos

\begin{tabular}{|c|c|c|c|c|c|}
\hline Peptidase/Inhibitor & Adult $>>$ Embryo $^{a}$ & Adult $=$ Embryo & Embryo $>>$ Adult $^{a}$ & Low expression $^{\text {b }}$ & Total \\
\hline Legumain & 9 & 1 & 0 & 9 & 19 \\
\hline Cystatin & 6 & 1 & 0 & 11 & 18 \\
\hline Cathepsin B & 15 & 4 & 0 & 5 & 24 \\
\hline Cathepsin L & 15 & 4 & 1 & 9 & 29 \\
\hline Calpain & 0 & 5 & 0 & 0 & 5 \\
\hline Caspase & 0 & 4 & 0 & 0 & 4 \\
\hline Thyropin & 0 & 4 & 1 & 0 & 5 \\
\hline Cathepsin O & 0 & 1 & 0 & 0 & 1 \\
\hline $\mathrm{GPI}$ & 0 & 1 & 0 & 0 & 1 \\
\hline Separase & 0 & 1 & 0 & 0 & 1 \\
\hline
\end{tabular}

${ }^{a}$ Number of genes with a FDR value $\leq 0.05$ and a fold change $\geq 2$.

${ }^{b}$ Number of genes expressed with a value $\leq 5$ in adults and embryos.

genomes of arthropods available so far, including ten insect species belonging to different orders, the crustacean D. pulex, and the acari I. scapularis. Gene expansions in C1A cysteine peptidases were also found, though in a lower extent, in insects such as the aphid A. pisum and the beetle $T$. castaneum, which also rely on cysteine peptidases for proteolytic digestion $[29,30]$. On the contrary, none of the insects analyzed belonging to the orders Diptera (D. melanogaster and A. gambiae), Lepidoptera (B. mori), Hymenoptera (C. floridanus, A. mellifera and $N$. vitripennis) and Phthiraptera (P. humanus), with a digestive system mostly based on serine peptidases [31], presented this extensive proliferation of cysteine peptidases. Hemipterans (R. prolixus) and ticks (I. scapularis), that in addition to cysteine peptidases use serine and/or aspartyl peptidases for proteolytic digestion [31], did not also have an expansion of their cysteine peptidases. In the case of the crustacean $D$. pulex, in which digestion commonly relies on trypsins and chymotrypsins [32], proliferation of cysteine peptidase genes could be associated to specific adaptations to the lifestyle of a planktonic filter feeder in a highly variable aquatic environment. Thus, we could conclude that, in general, extensive C1A cysteine peptidase duplications in arthropods are correlated to their corresponding diet and could be related to nutritional functions.

Large-scaled gene amplification for legumains is a striking feature restricted to T. urticae. Legumains, also called asparaginyl endopeptidases, have been involved in the degradation of host hemoglobin in ticks [9,33]. A legumain from Ixodes ricinus is located intracellularly in the vacuoles of gut epithelial cells where digestion occur throughout the whole duration of feeding [15], and contributes directly to the cleavage of hemoglobin and/or the processing of other gut peptidase zymogens [34]. In most acariform mites, digestion is considered to be composed of an extracellular phase achieved by enzymes secreted to the lumen of the midgut, followed by an intracellular one that can be performed in free floating cells $[2,3,35]$. Legumains could be involved in the intracellular phase by processing some other peptidases or by a direct action on the plant feed proteins. Large expansion of this family in $T$. urticae could be related to the broad range of $\mathrm{C} 1 \mathrm{~A}$ cysteine peptidases that need to be activated for digestion or to their direct role on host selectivity by processing plant toxic proteins.

Massive gene expression data have been previously used to detect different peptidases in the gut of insects [30,36-38] and ticks [39]. Since digestive proteolytic enzymes should be abundantly generated to deal with the higher volume of food that must be hydrolysed in free living developmental stages, genes involved in the proteolytic digestion are expected to be expressed at higher rate in free leaving stages comparing to embryos. Transcriptomic data analysis in T. urticae indicates that most cathepsin B and L peptidases and legumains are expressed at a higher rate in larvae, nymphs and adults, confirming their putative role in proteolytic digestion. On the contrary, other gene cysteine peptidase families like caspases, separases, calpains and GPI:protein transamidases have similar levels of expression in the four developmental stages analysed, supporting a role for these genes in some other endogenous processes across the whole life of the spider mite.

The activity of this extended number of peptidases must be regulated in the acari. Among cysteine peptidase inhibitors, the cystatin, thyropin and C1A cysteine peptidase propeptide families have been previously described in arthropods. Cysteine peptidase propeptides are inhibitory domains that are included in all the C1A peptidases analysed in this study. They have a conserved role in the control of $\mathrm{C} 1 \mathrm{~A}$ peptidase activity before the inhibitory domain is released and the peptidase becomes active [40]. The $T$. urticae genome does not contain small propeptide-like genes similar to those present in Bombyx [22]. 
Remarkably, the expansion in peptidase genes of the families $\mathrm{C} 1 \mathrm{~A}$ and $\mathrm{C} 13$ in T. urticae is accompanied by a proliferation of cystatin inhibitors putatively targeting both enzyme families. The physiological functions of this extended number of I25 cystatins in T. urticae may be related to their inhibitory activity on specific $\mathrm{C} 1 \mathrm{~A}$ and C13 peptidases. In insects, cystatins have been associated to processes related to the regulation of endogenous protease activity, such as insect morphogenesis and development $[23,41]$, and/or in the inhibition of heterologous cysteine peptidases, e.g. during insect immune response and plant feeding [21,42]. Moreover, cystatins have been previously related to blood-feeding in ticks $[27,43,44]$, where they are expressed in salivary glands and the midgut contributing to suppress host immune response. The fact that cystatins have evolved differentially in arthropod clades implies a specific function for the members of the proliferated groups in each clade. Thus, besides their potential implications in some other physiological processes, spider mite cystatins could have a role in mite feeding by regulating their own digestive cysteine peptidases, by inhibiting cysteine peptidases of the host after feeding, and/or by contributing to counteract host defence mechanisms. Several results support the putative involvement of cystatins in mite feeding: i) transcriptomic data analysis indicate that group two and four cystatins are consistently detected at greater levels in larvae, nymphs and adults relative to embryos; ii) the amino acid sequences for several spider mite cystatin groups have diverged deeply from consensus cystatin motifs related to cysteine peptidase inhibition. In fact, group 4 cystatin genes code for a new type of cystatins with no known paralogs in living organisms that putatively contain $\mathrm{C} 13$ peptidase binding domains, but do not contain canonical $\mathrm{C} 1 \mathrm{~A}$ peptidase binding domains. These results support an evolutionary scenario in which cystatin family may have evolved in the spider mite to control the proliferation of divergent $\mathrm{C} 1 \mathrm{~A}$ cysteine peptidases and C13 legumains involved in protein digestion. Alternatively, cystatins in T. urticae may have evolved as specific adaptations to the lifestyle of an extremely polyphagous species to deal with highly variable resources.

On the contrary, limited data on the physiological roles for thyropins are available. Thyropins are thyroglobulin domains capable of exhibiting inhibitory activity, which has been reported mainly against C1A cysteine peptidases [45]. No arthropod thyropins have been characterized to date, although some members are present in the sialotranscriptome of several ticks $[46,47]$. The possible implication of thyropins in the control of peptidases involved in feeding comes from the reduction of cysteine peptidase activity and deleterious effects on larval growth when the thyropin equistatin isolated from sea anemone Actinia equine was introduced in the diet of the insects $T$. castaneum and Leptinotarsa decemlineata $[48,49]$. However, the parallel evolution of thyropins, with complex architectures shared by different arthropod clades, and their transcriptional pattern, having most of them a similar level of expression in adults and embryos, suggest a common conserved role that could be in some cases related to feeding, but no specific for the spider mite nutritional aspects.

\section{Conclusions}

Comparative genomic analyses have provided valuable insights into the conservation and evolution of cysteine peptidases and their inhibitors in T. urticae. A phylogenetic analysis of these gene families in representative species of different arthropod taxonomic groups has allowed us to state that clade-specific proliferations are common to $\mathrm{C} 1 \mathrm{~A}$ papain-like and C13 legumain-like peptidases, as well as to the I25 cystatins, whereas the I31 thyropins are evolutionarily more conserved among arthropod clades. Extensive duplications and transcriptomic data for spider mite $\mathrm{C} 1 \mathrm{~A}$ and $\mathrm{C} 13$ peptidases support their role in proteolytic digestion. The expansion of the I25 cystatin family of inhibitors and their highest expression in feeding stages suggest a role for some cystatin members in mite feeding by regulating endogenous or exogenous peptidases and/or by contributing to counteract host defence mechanisms. In conclusion, mite-specific proliferations of both peptidases and their inhibitors are in accordance with mite's feeding features and support a key role for these proteins in allowing the broad plant host feeding range described for the two-spotted spider mite.

\section{Methods}

\section{Sequence searches}

Blast searches for cystatins, thyropins and cysteine peptidases were performed in publicly available genome databases. Sequences for Tetranychus urticae were obtained at the BOGAS (Bioinformatics Online Genome Annotation System) website [50]. Sequences for other arthopods were identified by searching the current genome releases at: the ant Fourmidable database [51]; the AphidBase [52]; the invertebrate vectors for human pathogens VectorBase [53]; the Daphnia wFleaBase [54]; the FlyBase [55]; the Bombyx mori SilkDB [56]; the BeeBase [57]; the wasp NasoniaBase [58]; and the BeetleBase [59]. Blast searches were made in a recurrent way. First, a complete amino acid arthropod sequence from data banks corresponding to a protein of the family was used. Then, the protein sequences of each arthropod species were used to search in the species. Finally, after an alignment of the proteins found in arthropods, the conserved region surrounding the catalytic sites from the species most related was used to a final search in each arthropod species. 
Information about gene models for all these proteins is compiled in Additional file 5.

\section{Domain architecture prediction}

Amino acid sequences for arthropod proteins putatively including at least one cystatin or thyropin domain were subjected to a sequence search in the Pfam database $\mathrm{v}$ 26.0 [60] to know the combination of domains within each protein. From these results, the domain architecture of each protein was manually schematized.

\section{Protein alignments and Phylogenetic trees}

Alignments of the amino acid sequences were performed using the default parameters of MUSCLE version 3.8 [61]. Sequences with extensive gaps were manually excluded from phylogenetic analysis using the multiple alignment editor Jalview version 2.7 [62]. Phylogenetic and molecular evolutionary analyses were conducted using the programs PhyML 3.0 and MEGA version 5.0 $[63,64]$. The program PROTTEST (2.4) was employed for selecting the model of protein evolution that fits better to each alignment according to the corrected Akaike Information Criterion [65]. The parameters of the selected models were employed to reconstruct the displayed clan $\mathrm{CD}$ cysteine peptidases trees by means of a maximum likelihood PhyML method using a BIONJ starting tree. The approximate likelihood-ratio test (aLRT) based on a Shimodaira-Hasegawa-like procedure was used as statistical test for non-parametric branch support [66]. All families were also analysed with the Maximum parsimony and the Neighbour-Joining algorithms, and with different gap penalties. No significant differences in the tree topologies were detected.

\section{Molecular modelling of $T$. urticae cystatins}

The three-dimensional structures of the T. urticae cystatins were modelled using the standard automated routine of SWISS-MODEL program [67]. The known crystal structures of the cystatins from chicken egg (PDB identifier 1YVBI), soft tick (3LOR), and human cystatin F $(2 \mathrm{CH} 9)$ were used to construct the homology-based models. The template structures were selected on the basis of highest sequence similarities. Models were evaluated with the QMEAN Z-score for predicting the absolute quality of a model [68]. The Swiss-PdbViewer program [69] was used to generate the single images of protein models.

\section{In silico transcriptome expression}

The transcriptomic information available at the BOGAS T. urticae website [50] was used to the developmental expression analyses. The protocol to normalized read counts of RNA-seq Illumina reads has been previously described [1]. To determine significant differences in the levels of gene expression between spider mite embryos and adults, we defined as differentially expressed genes that for which the false discovery rate (FDR) corrected p-value was $\leq 0.05$ and for which the fold change was $\geq 2$ (either up- or down-regulated).

\section{Additional files}

\begin{abstract}
Additional file 1: Alignments performed using the MUSCLE program of the amino acid sequences corresponding to the proteins used in this study. (A) Alignment of cystatin domains from selected arthropod species. (B) Alignment of thyropin domains from selected arthropod species. (C) Alignment of T. urticae cystatin domains.
\end{abstract}

Additional file 2: Complete phylogenetic trees of the cystatin (A) and thyropins (B) domains from selected arthropod species.

Additional file 3: Domain architectures of the proteins with thyropin domains from the selected arthropod species.

Additional file 4: Alignment of the different four groups of cystatin sequences from $T$. urticae showing conserved motifs and structural features.

Additional file 5: Information about gene models and accession numbers corresponding to the proteins (cystatins, thyropins, C1A peptidases and C13 peptidases) used in this study.

Competing interests

The authors declare that they have no competing interests.

\section{Acknowledgements}

The financial support from the Ministerio de Educación y Ciencia (AGL201123650) and Government of Canada through Genome Canada and the Ontario Genomics Institute (OGI-046) is gratefully acknowledged.

\section{Author details}

'Department of Biology WSC 339/341, The University of Western Ontario, 1151 Richmond St, London, ON N6A 5B7, Canada. ${ }^{2}$ Laboratorio de Interacción Planta-Insecto, Departamento de Biología Medioambiental, Centro de Investigaciones Biológicas, CSIC, Ramiro de Maeztu, 9, 28040, Madrid, Spain. ${ }^{3}$ Centro de Biotecnología y Genómica de Plantas, Universidad Politécnica de Madrid, Campus Montegancedo, 28223-Pozuelo de Alarcón, Madrid, Spain.

\section{Authors' contributions}

MES, PH-C, VG and MM carried out the sequence recovery and analysis. ID, FO, MG and MM designed the study and carried out the interpretation of the results. MM drafted the manuscript. All authors read and approved the final manuscript.

Received: 9 April 2012 Accepted: 11 July 2012

Published: 11 July 2012

\section{References}

1. Grbic M, Van Leeuwen T, Clark RM, Rombauts S, Rouze P, Grbic V, Osborne EJ, Dermauw W, Ngoc PC, Ortego F, et al: The genome of Tetranychus urticae reveals herbivorous pest adaptations. Nature 2011, 479(7374):487-492.

2. Filimonova SA: The ultrastructural investigation of the midgut in the quill mite Syringophilopsis fringilla (Acari, Trombidiformes: Syringophilidae). Arthropod Struct Dev 2009, 38(4):303-313.

3. Hamilton KA, Nisbet AJ, Lehane MJ, Taylor MA, Billingsley PF: A physiological and biochemical model for digestion in the ectoparasitic mite, Psoroptes ovis (Acari: Psoroptidae). Int J Parasitol 2003, 33(8):773-785.

4. Carrillo L, Martinez M, Ramessar K, Cambra I, Castanera P, Ortego F, Diaz I: Expression of a barley cystatin gene in maize enhances resistance against phytophagous mites by altering their cysteine-proteases. Plant Cell Rep 2011, 30(1):101-112.

5. Nisbet AJ, Billingsley PF: A comparative survey of the hydrolytic enzymes of ectoparasitic and free-living mites. Int J Parasitol 2000, 30(1):19-27. 
6. Rawlings ND, Barrett AJ, Bateman A: MEROPS: the database of proteolytic enzymes, their substrates and inhibitors. Nucleic Acids Res 2012, 40 (Database issue):D343-350.

7. Turk V, Stoka V, Vasiljeva O, Renko M, Sun T, Turk B, Turk D: Cysteine cathepsins: From structure, function and regulation to new frontiers. Biochim Biophys Acta 2012, 1824(1):68-88.

8. Cristofoletti PT, Ribeiro AF, Terra WR: The cathepsin L-like proteinases from the midgut of Tenebrio molitor larvae: sequence, properties, immunocytochemical localization and function. Insect Biochem Mol Biol 2005, 35(8):883-901.

9. Horn M, Nussbaumerova M, Sanda M, Kovarova Z, Srba J, Franta Z, Sojka D, Bogyo M, Caffrey CR, Kopacek $P$, et al: Hemoglobin digestion in bloodfeeding ticks: mapping a multipeptidase pathway by functional proteomics. Chem Biol 2009, 16(10):1053-1063

10. Soares-Costa A, Dias AB, Dellamano M, de Paula FF, Carmona AK, Terra WR, Henrique-Silva F: Digestive physiology and characterization of digestive cathepsin L-like proteinase from the sugarcane weevil Sphenophorus levis. J Insect Physiol 2011, 57(4):462-468.

11. Cho WL, Tsao SM, Hays AR, Walter R, Chen JS, Snigirevskaya ES, Raikhel AS: Mosquito cathepsin B-like protease involved in embryonic degradation of vitellin is produced as a latent extraovarian precursor. $J$ Biol Chem 1999, 274(19):13311-13321.

12. Liu J, Shi GP, Zhang WQ, Zhang GR, Xu WH: Cathepsin L function in insect moulting: molecular cloning and functional analysis in cotton bollworm, Helicoverpa armigera. Insect Mol Biol 2006, 15(6):823-834

13. Seixas A, Dos Santos PC, Velloso FF, Da Silva Vaz I Jr, Masuda A, Horn F, Termignoni C: A Boophilus microplus vitellin-degrading cysteine endopeptidase. Parasitology 2003, 126(Pt 2):155-163.

14. Uchida K, Ohmori D, Ueno T, Nishizuka M, Eshita Y, Fukunaga A, Kominami E: Preoviposition activation of cathepsin-like proteinases in degenerating ovarian follicles of the mosquito Culex pipiens pallens. Dev Biol 2001, 237 (1):68-78.

15. Franta Z, Frantova H, Konvickova J, Horn M, Sojka D, Mares M, Kopacek P. Dynamics of digestive proteolytic system during blood feeding of the hard tick Ixodes ricinus. Parasit Vectors 2010, 3:119.

16. Kordis D, Turk V: Phylogenomic analysis of the cystatin superfamily in eukaryotes and prokaryotes. BMC Evol Biol 2009, 9:266

17. Turk V, Bode W: The cystatins: protein inhibitors of cysteine proteinases. FEBS Lett 1991, 285(2):213-219.

18. Kotsyfakis M, Horka H, Salat J, Andersen JF: The crystal structures of two salivary cystatins from the tick Ixodes scapularis and the effect of these inhibitors on the establishment of Borrelia burgdorferi infection in a murine model. Mol Microbiol 2010, 77(2):456-470.

19. Alvarez-Fernandez M, Barrett AJ, Gerhartz B, Dando PM, Ni J, Abrahamson $M$ : Inhibition of mammalian legumain by some cystatins is due to a novel second reactive site. J Biol Chem 1999, 274(27):19195-19203.

20. Martinez M, Diaz-Mendoza M, Carrillo L, Diaz I: Carboxy terminal extended phytocystatins are bifunctional inhibitors of papain and legumain cysteine proteinases. FEBS Lett 2007, 581(16):2914-2918.

21. Buarque DS, Spindola LM, Martins RM, Braz GR, Tanaka AS: Tigutcystatin, a cysteine protease inhibitor from Triatoma infestans midgut expressed in response to Trypanosoma cruzi. Biochem Biophys Res Commun 2011, 413 (2):241-247.

22. Miyaji T, Murayama S, Kouzuma Y, Kimura N, Kanost MR, Kramer KJ, Yonekura M: Molecular cloning of a multidomain cysteine protease and protease inhibitor precursor gene from the tobacco hornworm (Manduca sexta) and functional expression of the cathepsin F-like cysteine protease domain. Insect Biochem Mol Biol 2010, 40(12):835-846.

23. Saito H, Suzuki T, Ueno K, Kubo T, Natori S: Molecular cloning of cDNA for sarcocystatin $A$ and analysis of the expression of the sarcocystatin $A$ gene during development of Sarcophaga peregrina. Biochemistry 1989, 28 (4):1749-1755.

24. Agarwala KL, Kawabata S, Hirata M, Miyagi M, Tsunasawa S, Iwanaga S: A cysteine protease inhibitor stored in the large granules of horseshoe crab hemocytes: purification, characterization, cDNA cloning and tissue localization. J Biochem 1996, 119(1):85-94

25. Novinec M, Kordis D, Turk V, Lenarcic B: Diversity and evolution of the thyroglobulin type-1 domain superfamily. Mol Biol Evol 2006, 23(4):744-755.

26. Yamamoto Y, Watabe S, Kageyama T, Takahashi SY: A novel inhibitor protein for Bombyx cysteine proteinase is homologous to propeptide regions of cysteine proteinases. FEBS Lett 1999, 448(2-3):257-260.
27. Kotsyfakis M, Karim S, Andersen JF, Mather TN, Ribeiro JM: Selective cysteine protease inhibition contributes to blood-feeding success of the tick Ixodes scapularis. J Biol Chem 2007, 282(40):29256-29263.

28. Martinez M: Plant protein-coding gene families: emerging bioinformatics approaches. Trends Plant Sci 2011, 16(10):558-567.

29. Rispe C, Kutsukake M, Doublet V, Hudaverdian S, Legeai F, Simon JC, Tagu $D$, Fukatsu T: Large gene family expansion and variable selective pressures for cathepsin B in aphids. Mol Biol Evol 2008, 25(1):5-17.

30. Morris K, Lorenzen MD, Hiromasa Y, Tomich JM, Oppert C, Elpidina EN, Vinokurov K, Jurat-Fuentes JL, Fabrick J, Oppert B: Tribolium castaneum larval gut transcriptome and proteome: $A$ resource for the study of the coleopteran gut. J Proteome Res 2009, 8(8):3889-3898.

31. Terra WR, Ferreira C: 4.5 - Biochemistry of Digestion. In Comprehensive Molecular Insect Science. Edited by Lawrence IG, Kostas I, Sarjeet SG. Amsterdam: Elsevier; 2005:171-224.

32. Schwerin S, Zeis B, Lamkemeyer T, Paul RJ, Koch M, Madlung J, Fladerer C, Pirow R: Acclimatory responses of the Daphnia pulex proteome to environmental changes. II. Chronic exposure to different temperatures (10 and 20 degrees C) mainly affects protein metabolism. BMC Physiol 2009, 9:8.

33. Alim MA, Tsuji N, Miyoshi T, Islam MK, Hatta T, Yamaji K, Fujisaki K. Developmental stage- and organ-specific expression profiles of asparaginyl endopeptidases/legumains in the ixodid tick Haemaphysalis longicornis. J Vet Med Sci 2008, 70(12):1363-1366.

34. Sojka D, Hajdusek O, Dvorak J, Sajid M, Franta Z, Schneider EL, Craik CS, Vancova M, Buresova $V$, Bogyo $M$, et al: IrAE: an asparaginyl endopeptidase (legumain) in the gut of the hard tick Ixodes ricinus. Int $J$ Parasitol 2007, 37(7):713-724.

35. Mothes-Wagner U: Fine structure of the 'hindgut' of the two-spotted spider mite, Tetranychus urticae, with special reference to origin and function. Exp Appl Acarol 1985, 1(3):253-272.

36. Dostalova A, Votypka J, Favreau AJ, Barbian KD, Volf P, Valenzuela JG, Jochim RC: The midgut transcriptome of Phlebotomus (Larroussius) perniciosus, a vector of Leishmania infantum: comparison of sugar fed and blood fed sand flies. BMC Genomics 2011, 12:223.

37. Jochim RC, Teixeira CR, Laughinghouse A, Mu J, Oliveira F, Gomes RB, Elnaiem DE, Valenzuela JG: The midgut transcriptome of Lutzomyia longipalpis: comparative analysis of cDNA libraries from sugar-fed, blood-fed, post-digested and Leishmania infantum chagasi-infected sand flies. BMC Genomics 2008, 9:15.

38. Zhang S, Shukle R, Mittapalli O, Zhu YC, Reese JC, Wang H, Hua BZ, Chen MS: The gut transcriptome of a gall midge, Mayetiola destructor. J Insect Physiol 2010, 56(9):1198-1206

39. Anderson JM, Sonenshine DE, Valenzuela JG: Exploring the mialome of ticks: an annotated catalogue of midgut transcripts from the hard tick, Dermacentor variabilis (Acari: Ixodidae). BMC Genomics 2008, 9:552.

40. Wiederanders B, Kaulmann G, Schilling K: Functions of propeptide parts in cysteine proteases. Curr Protein Pept Sci 2003, 4(5):309-326.

41. Goto SG, Denlinger DL: Genes encoding two cystatins in the flesh fly Sarcophaga crassipalpis and their distinct expression patterns in relation to pupal diapause. Gene 2002, 292(1-2):121-127.

42. Francischetti IM, Lopes AH, Dias FA, Pham VM, Ribeiro JM: An insight into the sialotranscriptome of the seed-feeding bug, Oncopeltus fasciatus. Insect Biochem Mol Biol 2007, 37(9):903-910.

43. Grunclova L, Horn M, Vancova M, Sojka D, Franta Z, Mares M, Kopacek P. Two secreted cystatins of the soft tick Ornithodoros moubata: differential expression pattern and inhibitory specificity. Biol Chem 2006, 387 (12):1635-1644.

44. Zhou J, Liao M, Ueda M, Gong H, Xuan X, Fujisaki K: Characterization of an intracellular cystatin homolog from the tick Haemaphysalis longicornis. Vet Parasitol 2009, 160(1-2):180-183.

45. Mihelic M, Turk D: Two decades of thyroglobulin type-1 domain research. Biol Chem 2007, 388(11):1123-1130.

46. Anatriello E, Ribeiro JM, de Miranda-Santos IK, Brandao LG, Anderson JM Valenzuela JG, Maruyama SR, Silva JS, Ferreira BR: An insight into the sialotranscriptome of the brown dog tick Rhipicephalus sanguineus. RBMC Genomics 2010, 11:450.

47. Francischetti IM, Sa-Nunes A, Mans BJ, Santos IM, Ribeiro JM: The role of saliva in tick feeding. Front Biosci 2009, 14:2051-2088.

48. Gruden K, Strukelj B, Popovic T, Lenarcic B, Bevec T, Brzin J, Kregar I, HerzogVelikonja J, Stiekema WJ, Bosch D, et al: The cysteine protease activity of 
Colorado potato beetle (Leptinotarsa decemlineata Say) guts, which is insensitive to potato protease inhibitors, is inhibited by thyroglobulin type-1 domain inhibitors. Insect Biochem Mol Biol 1998, 28(8):549-560.

49. Oppert B, Morgan TD, Hartzer K, Lenarcic B, Galesa K, Brzin J, Turk V, Yoza K, Ohtsubo K, Kramer KJ: Effects of proteinase inhibitors on digestive proteinases and growth of the red flour beetle, Tribolium castaneum (Herbst) (Coleoptera: Tenebrionidae). Comp Biochem Physiol C Toxicol Pharmacol 2003, 134(4):481-490.

50. Tetranychus urticae website: BOGAS (Bioinformatics Online Genome Annotation System). http://bioinformaticspsbugentbe/webtools/bogas/ overview/Tetur.

51. The ant Fourmidable database. http://antgenomesorg/.

52. The AphidBase. http://wwwaphidbasecom/aphidbase/.

53. The invertebrate vectors for human pathogens VectorBase. http:// wwwvectorbaseorg/

54. The Daphnia wFleaBase. http://wfleabaseorg/.

55. The FlyBase. http://flybaseorg/.

56. The Bombyx mori SilkDB. http://silkwormgenomicsorgcn/.

57. The BeeBase. http://hymenopteragenomeorg/beebase/

58. The wasp NasoniaBase. http://hymenopteragenomeorg/nasonia/.

59. The BeetleBase. http://wwwbeetlebaseorg/.

60. Punta M, Coggill PC, Eberhardt RY, Mistry J, Tate J, Boursnell C, Pang N, Forslund K, Ceric G, Clements J, et al: The Pfam protein families database. Nucleic Acids Res 2012, 40(Database issue):D290-301.

61. Edgar RC: MUSCLE: multiple sequence alignment with high accuracy and high throughput. Nucleic Acids Res 2004, 32(5):1792-1797.

62. Waterhouse AM, Procter JB, Martin DM, Clamp M, Barton GJ: Jalview Version 2-a multiple sequence alignment editor and analysis workbench. Bioinformatics 2009, 25(9):1189-1191.

63. Guindon S, Dufayard JF, Lefort V, Anisimova M, Hordijk W, Gascuel O: New algorithms and methods to estimate maximum-likelihood phylogenies: assessing the performance of PhyML 3.0. Syst Biol 2010, 59(3):307-321.

64. Tamura K, Peterson D, Peterson N, Stecher G, Nei M, Kumar S: MEGA5: molecular evolutionary genetics analysis using maximum likelihood, evolutionary distance, and maximum parsimony methods. Mol Biol Evol 2011, 28(10):2731-2739.

65. Abascal F, Zardoya R, Posada D: ProtTest: selection of best-fit models of protein evolution. Bioinformatics 2005, 21(9):2104-2105.

66. Anisimova M, Gil M, Dufayard JF, Dessimoz C, Gascuel O: Survey of branch support methods demonstrates accuracy, power, and robustness of fast likelihood-based approximation schemes. Syst Biol 2011, 60(5):685-699.

67. Bordoli L, Kiefer F, Arnold K, Benkert P, Battey J, Schwede T: Protein structure homology modeling using SWISS-MODEL workspace. Nat Protoc 2009, 4(1):1-13.

68. Benkert $P$, Biasini M, Schwede T: Toward the estimation of the absolute quality of individual protein structure models. Bioinformatics 2011, 27 (3):343-350.

69. Guex N, Diemand A, Peitsch MC: Protein modelling for all. Trends Biochem Sci 1999, 24(9):364-367.

\section{Submit your next manuscript to BioMed Central and take full advantage of:}

- Convenient online submission

- Thorough peer review

- No space constraints or color figure charges

- Immediate publication on acceptance

- Inclusion in PubMed, CAS, Scopus and Google Scholar

- Research which is freely available for redistribution

Submit your manuscript at www.biomedcentral.com/submit
C Biomed Central 